\title{
Observation of Negative Refraction and Negative Phase Velocity in True Left-Handed Metamaterials
}

\author{
Ekmel Ozbay ${ }^{1}$ and Costas M. Soukoulis ${ }^{2}$ \\ ${ }^{1}$ Department of Physics, Department of Electrical and Electronics Engineering, Nanotechnology Research \\ Center, Bilkent University, Bilkent, 06800 Ankara, Turkey \\ ${ }^{2}$ Institute of Electronic Structure and Laser, Foundation for Research and Technology, Hellas \& Dept. of \\ Materials Science and Technology, University of Crete, Greece
}

\begin{abstract}
We report a true left-handed (LH) behavior in a composite metamaterial consisting of periodically arranged split ring resonator (SRR) and wire structures. The magnetic resonance of the SRR structure is demonstrated by comparing the transmission spectra of SRRs with that of closed SRRs. We confirmed experimentally that the effective plasma frequency of the LH material composed of SRRs and wires is lower than the plasma frequency of the wires. A well-defined left-handed transmission band with a peak value of $-1.2 \mathrm{~dB}(-0.3 \mathrm{~dB} / \mathrm{cm})$ is obtained. We also report the transmission characteristics of a $2 \mathrm{D}$ composite metamaterial (CMM) structure in free space. At the frequencies where left-handed transmission takes place, we experimentally confirmed that the CMM structure has effective negative refractive index. Phase shift between consecutive numbers of layers of CMM is measured and phase velocity is shown to be negative at the relevant frequency range. Refractive index values obtained from the refraction experiments and the phase measurements are in good agreement. The experimental results agree extremely well with the theoretical calculations.
\end{abstract}

Index Terms - Left-handed medium, metamaterial, negative phase velocity, negative refraction, split ring resonator.

\section{INTRODUCTION}

Veselago predicted that a medium with negative permittivity, $\varepsilon$, and negative permeability, $\mu$, will exhibit negative indices of refraction [1]. When both the permittivity and the permeability are negative the electric field, the magnetic field and the wavevector components form a lefthanded coordinate system, hence the name left-handed material (LHM) is used for description. Such a medium is expected to exhibit unusual physical properties such as negative refraction, reversal of Doppler shift, backward Cherenkov radiation. A medium with $\varepsilon<0$ can easily be realized, e.g. by periodically arranged metallic wires [2]. On the other hand, the $\mu(\omega)<0$ was a challenge due to lack of magnetic charge. Pendry et. al suggested that a periodic array of metallic split ring resonator (SRR) structures exhibit $\mu(\omega)$ $<0$ close to magnetic resonance frequency $\omega_{\mathrm{p}}$ [3]. This proposal brought the possibility of observing left-handed medium in to reality. Recently, this idea is brought to experimental investigation by constructing a composite metamaterial (CMM) consisting of two components which have $\varepsilon(\omega)<0$, a periodically arranged wire medium, and $\mu(\omega)<0, \quad$ a periodically arranged SRR medium, simultaneously over a certain frequency range, respectively [4],[5]. Various studies employing different structure designs extended this investigation [4]-[8]. Negative refraction of electromagnetic waves at the interface of CMMs is also observed [9]-[11] which supported the existence of a LHM. The parameters $\varepsilon$ and $\mu$ can be obtained by a retrieval procedure from the numerically calculated transmission and reflection data for finite size CMMs [12]-[14], under the assumption of a homogeneous medium. This procedure confirmed [15],[16] that a medium composed of SRRs and wires can indeed be characterized by effective $\varepsilon$ and $\mu$.

A medium transmits electromagnetic waves when both $\varepsilon$ and $\mu$ have the same sign, i.e., both are negative or both are positive. If they have opposite signs the medium effectively reflects the incoming electromagnetic wave. The existence of a pass band for the CMM within the respective stop bands of SRR-only and wire-only mediums is intuitively considered as evidence for $\mathrm{LH}$ behavior. We experimentally demonstrate that the dielectric response of the CMM differs substantially from that of the wire-only medium, by measuring $\omega_{\mathrm{p}}$ for the CMM and wire-only structures. It is evident that a shift in the plasma frequency renders the aforementioned intuitive approach inapplicable. This is a new feature that was not recognized in previous work. Based on this observation we present a new CMM structure which exhibits true left-handed behavior and has a transmission band with a peak value at $1.2 \mathrm{~dB}$. To our knowledge, this is the highest transmission value reported for a LHM.

\section{TRue LeFT-HANDED Composite Metamaterials}

In general, a SRR structure exhibits both magnetic response [3] induced by the solenoidal currents flowing around the SRR, and electric response [17], [18] by the dipole-like charge distribution along the incident electric field. The magnetic response of the SRR structure exhibits a resonance in the transmission spectrum. This resonance behavior is observed as a dip in the transmission spectrum of a single SRR structure. When SRRs are arranged in a periodic medium, due to the interaction between SRRs the resulting 
medium exhibits a band gap in the transmission spectrum. A bandgap in the transmission spectrum of periodic SRR medium may be due to negative $\varepsilon$ or negative $\mu$ or solely due to the periodicity. This ambiguity can be resolved by using a structure in which the splits in the ring resonators are closed (CSRR). This will destroy the magnetic resonance but still keep the electric resonance. A gap present in SRR but not in CSRR will then correspond to $\mu<0$.
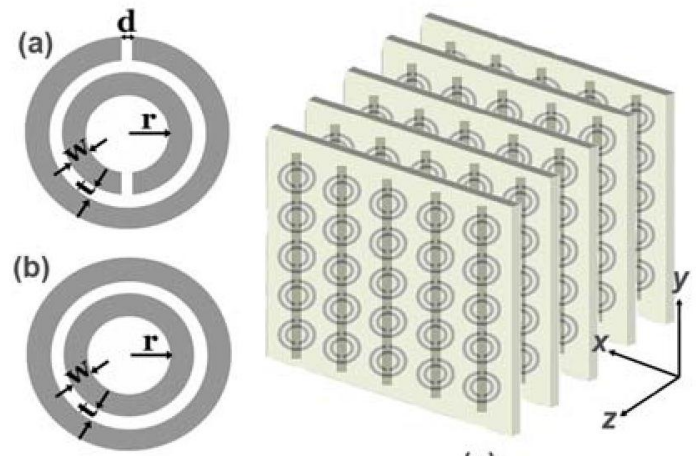

(c)

Fig. 1. (a) split ring resonator (SRR) (b) ring resonator with splits closed (CSRR) (c) Periodic CMM composed of SRRs on one side, wires on the other side of dielectric boards.

We fabricated SRR (Fig. 1.a) and CSRR (Fig. 1.b) structures by using standard printed circuit board technology. The measurement setup consists of HP $8510 \mathrm{C}$ vector network analyzer and standard gain horn antennas for transmitting and receiving electromagnetic waves. The incident field propagates along the $x$ direction with $E$, and $H$, along $y$ and $z$. directions, respectively.

The measured transmission spectrum for SRR (solid line) medium and the CSRR (dashed line) medium are shown in Fig. 2. The first band gap $(3.55-4.05 \mathrm{GHz})$ of the SRR medium is not present in the CSRR medium, indicating $\mu<0$. Note that the second band gap, 8.1-11.9 GHz, is observed both in the transmission spectrum of the SRR and CSRR mediums. This measurement clearly shows that the stop bands of an SRR medium can not be automatically attributed to "negative $\mu$ " behavior. Some of the observed gaps could also originate from the electrical response of the SRRs or from Bragg gaps due to periodicity.

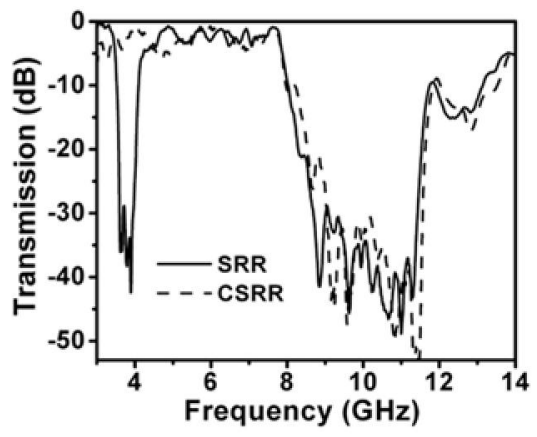

Fig. 2. Measured transmission spectra of a periodic SRR medium (solid) and periodic CSRR medium (dashed) between 3-14 GHz.

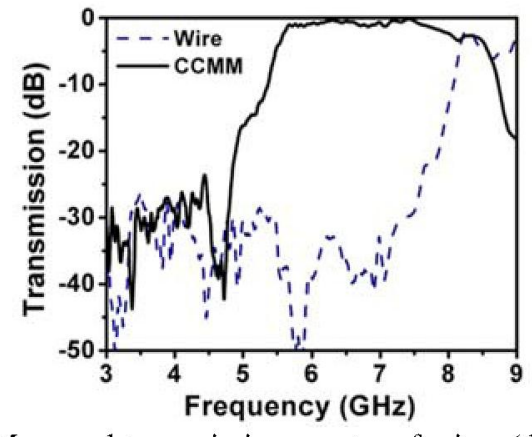

Fig. 3. Measured transmission spectra of wires (dashed line) and closed CMM (solid line) composed by arranging closed SRRs and wires periodically.

Another point to be discussed is the electric response of the CMM. Previously reported transmission results did not emphasize the interaction between SRR and wire structures. However, it was recently found [17] that the SRRs, in addition to their resonant magnetic response at $\omega_{m}$, exhibit a resonant electric response at $\omega_{0}$. The behavior is similar to that of a periodic cut-wire medium (wires of finite length) which exhibits a stop band with a well-defined lower edge due to the discontinuous wire geometry [8]. As a result, the SRRs contribute to the effective permittivity of the CMM, causing a downward shift on the plasma frequency determined solely from wire structures [17]. To demonstrate this effect, a CMM consisting of periodic alternating layers of CSRRs and wires is used (Fig. 1.c). Thickness, length and the width of the wires are $30 \mu \mathrm{m}, 13.5 \mathrm{~cm}$ and $0.9 \mathrm{~mm}$ respectively. Fig. 3 displays the measured transmission spectra of wire only medium and CMM consisting of CSRR and wire layers. The $\omega_{\mathrm{p}}$ of the wire-only structure around 8 $\mathrm{GHz}$, is reduced down to $5.3 \mathrm{GHz}$ within the closed CMM structure. As seen in Fig. 3, $\omega_{\mathrm{p}}$ of the CMM is lower than that of the wire-only medium alone. It is crucial to determine whether the shift in plasma cut-off frequency covers the magnetic resonance gap, which would render the CMM as a right-handed medium.

The experimental data presented in Fig. 3 clearly shows that one needs to take the shift in the plasma into account when designing mediums for LH behavior. To observe true LH behavior we designed the SRRs such that the first bandgap of SRR structure between $3.55-4.05 \mathrm{GHz}$ is not obscured by the shift in plasma frequency. The CMM structure is made of $N_{x}=5, N_{y}=15$, and $N_{z}=24$ unit cells. Each unit cell has a single SRR, and a copper wire from stacked SRR and wire layers, with lattice spacing $a_{x}=a_{y}=8.8$ $\mathrm{mm}, a_{z}=6.5 \mathrm{~mm}$. The transmission spectra for SRR only (solid line), wire only (dashed line) and CMM (bold solid line) periodic structures are displayed in Fig. 4. The simulations agree very well with the experimental data.

The CMM structure allows propagation of EM waves between 3.6 and $4.1 \mathrm{GHz}$, where both $\varepsilon$ and $\mu$ are negative. The CMM pass band exactly coincides with the stop band of SRR. The transmission peak at $3.9 \mathrm{GHz}$ is $-1.2 \mathrm{~dB}$, which is a significantly high value for a material made of metals. 


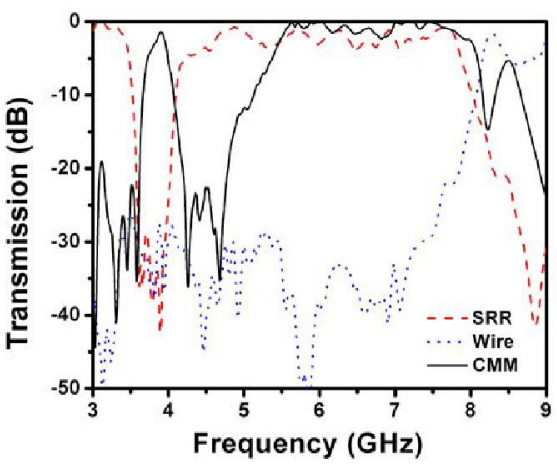

Fig. 4. Comparison of the measured transmission spectra of SRR (dashed), wire (dotted) and CMM solid line.

Similarly, the simulations predict a transmission peak at 3.92 $\mathrm{GHz}$ of $-1.5 \mathrm{~dB}$. We stress that, a similar transmission band is not present for a CMM composed of CSRRs and wires (Fig. 3). The electric response contribution of SRRs is also evident here: If the $\omega_{p}$ of the wire-only structure (dashed line in Fig. 4) were used to identify the $\varepsilon<0$ regime for the CMM, the transmission between $5.3-8 \mathrm{GHz}$ would have occured in a regime with $\varepsilon<0$ and $\mu>0$, which is not possible. However, as Fig. 3 suggests, the $\varepsilon>0$ regime of the combined electric response of SRRs and wires start at $5.3 \mathrm{GHz}$.

The transmitted phase of CMMs is measured to investigate the phase velocity within both the left-handed (3.73-4.05 $\mathrm{GHz}$ ) and right-handed (5.4-7.0 GHz) transmission bands. Phase measurements are performed on rectangular slabs of CMMs, with various numbers of layers. Fig. 5 shows the transmitted phase of CMMs (with varying number of layers) between frequencies 5.4-7.0 $\mathrm{GHz}$, where CMM acts as a right-handed medium. As shown in Fig. 5, the phase of the transmitted EM wave increases, when a longer CMM is used, which is a typical right-handed behavior. On the other hand, increasing the number of layers decreases the phase of the transmitted EM wave at the left-handed frequency region (Fig. 6). As shown in the inset of Fig. 6, the average phase shift is negative for the relevant frequency range, which indicates that the phase velocity is negative.

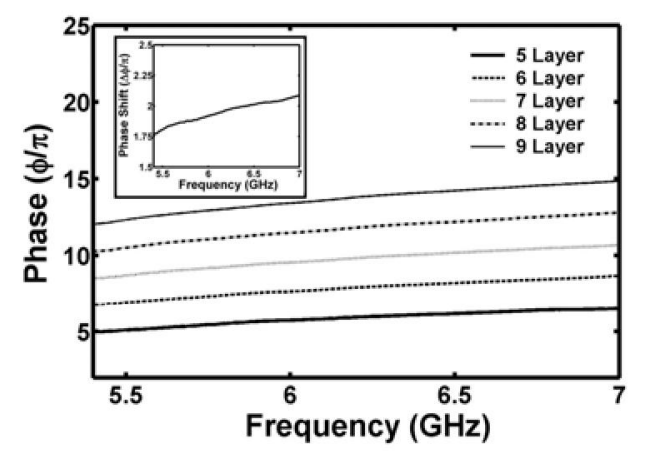

Fig. 5. Unwrapped transmission phase data obtained from different lengths of CMM between $5.4-7.0 \mathrm{GHz}$, where righthanded transmission peak takes place. Inset: Average phase difference between consecutive numbers of layers of CMM. Phase shift is positive between $5.4-7.0 \mathrm{GHz}$.

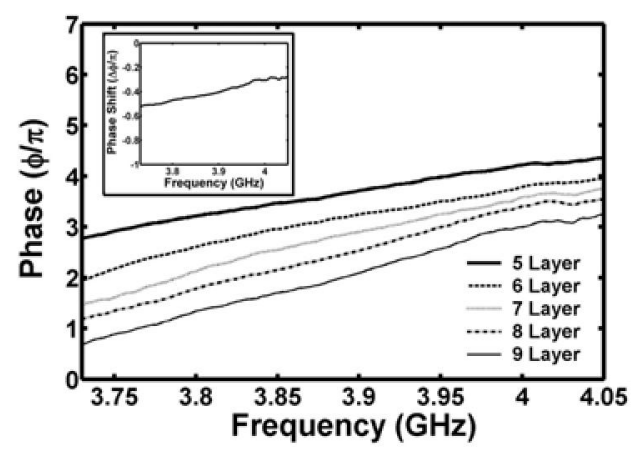

Fig. 6. Unwrapped transmission phase data obtained from different lengths of CMM between $3.73-4.05 \mathrm{GHz}$, where lefthanded transmission peak takes place. Inset: Average phase difference between consecutive numbers of layers of CMM. Phase shift is negative between $3.73-4.05 \mathrm{GHz}$.

\section{NEGATIVE REFRACTION}

Up to this point we concentrated on the transmission properties of metamaterial mediums consisting of SRR and wire structures. We demonstrated that a suitable combination of wires and SRRs exhibit true left-handed transmission peak in the transmission spectrum. Two very interesting properties of the LH medium are the negative refraction and the negative phase velocities. To investigate the negative refraction and negative phase velocities we studied the refraction and transmission phase properties of a 2 dimensional CMM medium.

The 2D CMM medium is fabricated by standard printed circuit board technology. The SRR and wire patterns are fabricated on the front and back sides of FR4 circuit boards which have $30 \mu \mathrm{m}$ thick deposited copper layer. The incident electromagnetic (EM) wave propagates along the $x$ direction, while $\mathbf{E}$ is along $y$ direction, and $\mathbf{H}$ is along $z$ direction.

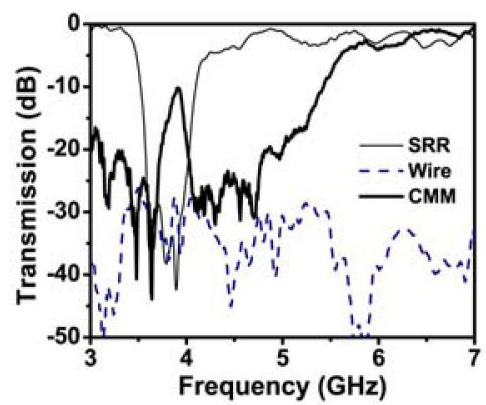

Fig. 7. Measured transmission spectra of a periodic SRR medium (solid line), periodic wire medium (dashed line) and 2D CMM medium (bold solid line) between 3-7 GHz.

Fig. 7 shows the measured transmission spectra of periodic SRRs (solid line) and wires (dashed line) and 2D CMM between 3-7 GHz. The bandgap of SRR between 3.55-4.05 $\mathrm{GHz}$ is due to magnetic resonance of periodic SRR medium, hence $\mu(\omega)<0$ for this frequency range. The 2D CMM structure allows propagation of EM waves between 3.7 and 
4.1 GHz, where both $\varepsilon$ and $\mu$ are negative. The CMM pass band coincides with the stop band of SRR. The transmission peak at $3.92 \mathrm{GHz}$ is $-10.2 \mathrm{~dB}$, which is significantly higher than the previously reported 2D CMM structures [5],[10]. The transmission band starting from $5.3 \mathrm{GHz}$ is due to downward plasma frequency shift, since the $\varepsilon>0$ regime of the combined electric response of SRRs and wires starts at $5.3 \mathrm{GHz}$.

We constructed a prism shaped 2D CMM structure (with a wedge angle of $\theta=26^{\circ}$ ) for negative refraction. Fig. 8 shows the transmission spectrum as a function of frequency and refraction angle. The transmitted EM waves are refracted towards the negative side of the surface normal within the frequency range of $3.73-4.05 \mathrm{GHz}$. At lower frequencies the EM waves are refracted at higher negative refraction angles, which results in a higher negative refractive index. On the other hand the refraction index is lowered with increasing frequencies. By employing Snell's law $\left(n_{C M M} \sin \theta_{i}\right.$ $=n_{\text {air }} \sin \theta_{r}$ ) an effective refractive index can be defined for the CMM. At $f=3.92 \mathrm{GHz}$, the incidence angle is $\theta_{i}=26^{\circ}$, and EM wave is refracted at an angle of $\theta_{r}=55^{\circ}$, then from Snell's law we obtain $n_{\text {eff }}=-1.87 \pm 0.05$ at $3.92 \mathrm{GHz}$.

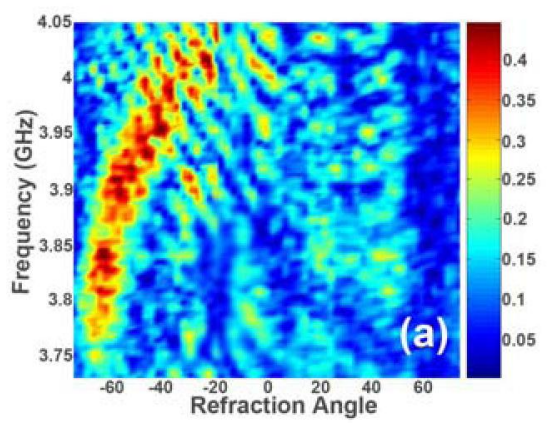

Fig. 8. Transmission spectra as a function of frequency and refraction angle.

\section{CONCLUSION}

In conclusion, we have successfully demonstrated true lefthanded behavior in free space with a high transmission peak. The left-handed transmission band exactly coincides with the region where both dielectric permittivity and magnetic permeability take negative values. In addition, we have successfully demonstrated a left-handed transmission band for 2D CMM structure in free space with a high transmission peak. We experimentally confirmed that 2D CMM has negative refractive index at the entire left-handed frequency range (3.73-4.05 $\mathrm{GHz})$. Phase shift and therefore phase velocity is shown to be negative, and the values of negative refractive indices obtained from the refraction experiments and the phase measurements are in good agreement.

\section{ACKNOWLEDGEMENT}

This work is supported by EU-DALHM, EUMETAMORPHOSE, EU-PHOREMOST.

\section{REFERENCES}

[1] V.G. Veselago, "The electrodynamics of substances with simultaneously negative values of permittivity and permeability," Sov. Phys. USPEKHI, vol. 10, p. 509, 1968.

[2] J.B. Pendry, A.J. Holden, D.J. Robbins, and W.J. Stewart, "Low frequency plasmons in thin-wire structures," Journal of Physics: Condensed Matter, vol. 10, p. 4785, 1998.

[3] J.B. Pendry, A.J. Holden, D.J. Robbins, and W.J. Stewart, "Magnetism from conductors and enhanced nonlinear phenomena," IEEE Trans. Microw. Th. Tech., vol. 47, p. 2075, 1999.

[4] D.R. Smith, W.J. Padilla, D.C. Vier, S.C. Nemat-Nasser, and S Schultz, "Composite medium with simultaneously negative permeability and permittivity," Phys. Rev. Lett., vol. 84, p. 4184,2000

[5] S. Linden, C. Enkrich, M. Wegener, J.F. Zhou, T. Koschny, and C.M. Soukoulis, "Magnetic response of metamaterials at 100 terahertz," Science, vol. 306, p. 1351, 2004.

[6] M. Bayindir, K. Aydin, E. Ozbay, P. Markos, and C.M. Soukoulis, "Transmission properties of composite metamaterial in free space," Appl. Phys. Lett., vol. 81, p. 120, 2002.

[7] N. Fang, H. Lee, C. Sun, and X. Zhang, "Sub-diffractionlimited optical imaging with a silver superlens," Science, vol. 308, p. 534, 2005.

[8] E. Ozbay, K. Aydin, E. Cubukcu, and M. Bayindir, "Transmission and Reflection Properties of Composite Double Negative Metamaterials in Free Space," IEEE Trans. Antennas Propag., vol. 51, p. 2592, 2003.

[9] I. Bulu, H. Caglayan, and E. Ozbay, "Experimental demonstration of subwavelength focusing of electromagnetic waves by labyrinth-based two-dimensional metamaterials," Optics Letters, vol. 31, p. 814, 2006.

[10] C.G. Parazzoli, R.B. Greegor, K. Li, B.E.C. Koltenbah, and M. Tanielian, "Experimental Verification and Simulation of Negative Index of Refraction Using Snell's Law," Phys. Rev. Lett., vol. 90, p. 107401, 2003.

[11] A.A. Houck, J.B. Brock, and I.L. Chuang, "Experimental Observations of a Left-Handed Material That Obeys Snell's Law," Phys. Rev. Lett., vol. 90, p. 137401, 2003.

[12] M. Kafesaki, T. Koschny, R. S. Penciu, T.F. Gundogdu, E.N Economou, and C.M. Soukoulis, "Left-handed metamaterials: detailed numerical studies of the transmission properties," J. of Optics A: Pure and Applied Optics, vol. 7, p. S12, 2005.

[13] P. Markos and C.M. Soukoulis, "Transmission properties and effective electromagnetic parameters of double negative metamaterials," Optics Express, vol. 11, 649 (2003).

[14] T. Koschny, P. Markos, E.N. Economou, D.R. Smith, and C.M. Soukoulis, "Impact of inherent periodic structure on effective medium description of left-handed and related metamaterials," Physical Review B, vol. 71, p. 245105, 2005.

[15] D.R. Smith and S. Schultz, P. Markos and C.M. Soukoulis, "Determination of effective permittivity and permeability of metamaterials from reflection and transmission coefficients," Phys. Rev. B, vol. 65, p. 195104, 2002.

[16] T. Koschny, P. Markos, D.R. Smith, and C.M. Soukoulis, "Resonant and antiresonant frequency dependence of the effective parameters of metamaterials," Phys. Rev. E, vol. 68, p. $065602(\mathrm{R}), 2003$.

[17] T. Koschny, M. Kafesaki, E.N. Economou, and C.M Soukoulis, "Effective medium theory of left-handed materials," Phys. Rev. Lett., vol. 93, p. 107402, 2004.

[18] K. Aydin, K. Guven, Lei Zhang, M. Kafesaki, C.M. Soukoulis, and E. Ozbay, "Experimental Observation of True Left-Handed Transmission Peak in Metamaterials," Optics Letters, vol. 29, p. 2623, 2004. 